\title{
Serviços e desserviços ambientais associados à agricultura urbana e periurbana no município de São Paulo
}

Bruno do N. Macedoa, Fernanda N. Comasa, Amarilis L. C. F. Gallardoa,b,*

a Escola Politécnica da Universidade de São Paulo, Av. Prof. Luciano Gualberto, Travessa do Politécnico, 380, São Paulo, Brasil, 05508-010.

• Universidade Nove de Julho, Programa de Pós-Graduação em Cidades Inteligentes e Sustentáveis, R. Vergueiro, 1289, São Paulo, Brasil, 01504-001.

\begin{abstract}
Informações
Recebido 17 Maio 2021

Aceito 29 Junho 2021

Palavras-chave

Agricultura Urbana e

Periurbana

Segurança Alimentar

Serviços Ambientais

Planejamento Urbano

Políticas Públicas
\end{abstract}

\section{Resumo}

A Agricultura Urbana e Periurbana (AUP) representa uma importante produção agrícola nas zonas periféricas urbanas, contribuindo para ampliar a segurança alimentar, resiliência urbana e renda. A AUP também associa serviços e desserviços ambientais que devem ser considerados no planejamento urbano. O objetivo deste trabalho é discutir sobre os principais serviços e desserviços ambientais associados à agricultura urbana e periurbana no município de São Paulo, cujo Plano Diretor Estratégico incentiva essa prática. O método da pesquisa usa revisão sistemática da literatura para a base conceitual dos serviços ecossistêmicos e análise espacial de dados georreferenciados de bases públicas para compor um mapeamento das iniciativas de AUP no município. Foram identificados 12 serviços ambientais e 5 desserviços ambientais, além de 197 iniciativas em áreas onde há um incentivo à prática, mas que deveriam ser protegidas de ações antrópicas. Conclui-se que a AUP tem potencial de oferecer benefícios ao ambiente, ainda que possa exercer pressões negativas ao ambiente.

\section{Environmental services and disservices associated with urban and periurban agriculture in the city of São Paulo}

\section{Article info \\ Received 17 May 2021 \\ Accepted 29 June 2021}

\section{Keywords}

Urban and Periurban

Agriculture

Food Security

Environmental Services

Urban Planning

Public Policy

\begin{abstract}
Urban and Periurban Agriculture (UPA) represents an important agricultural production in peripheral urban areas, contributing to increase food security, urban resilience and income. The UPA also associates environmental services and disservices that must be considered in urban planning. The objective of this work is to discuss the main environmental services and disservices associated with urban and periurban agriculture in São Paulo, which was chosen because its Strategic Master Plan encourages this practice. The research method comprises a systematic literature review for the conceptual basis of ecosystem services and spatial analysis of georeferenced data from public databases to compose a mapping of UPA initiatives in São Paulo. 12 environmental services and 5 environmental disservices associated with the AUP were identified in São Paulo, besides 197 initiatives in areas where there is an incentive, but also that should be protected from human actions. Concluding, UPA has the potential to offer environmental benefits, although it can exert negative pressures on the environment.
\end{abstract}

\section{Servicios y perjuicios ambientales asociados a la agricultura urbana y periurbana en la ciudad de São Paulo}

\section{Información}

Recibido 17 Mayo 2021

Aceptado 29 Junio 2021

\section{Palabras clave}

Agricultura Urbana y

Periurbana

Seguridad alimentaria

Servicios ambientales

Planificación urbana

Políticas públicas

\section{Resumen}

La Agricultura Urbana y Periurbana (AUP) representa una importante producción agrícola en las zonas urbanas periféricas, contribuyendo a incrementar la seguridad alimentaria, resiliencia urbana y ingresos. La AUP también asocia servicios y perjuicios ambientales que deben ser considerados en el planeamiento urbano. El objetivo de este trabajo es discutir los principales servicios y perjuicios ambientales asociados a la agricultura urbana y periurbana existentes en São Paulo, que fue elegida porque su Plan Maestro fomenta esta práctica. El método de investigación utiliza una revisión sistemática de la literatura para la base conceptual de los servicios ecosistémicos y el análisis espacial de datos georreferenciados de bases de datos públicas para componer un mapeo de las iniciativas de AUP en São Paulo. Se identificaron 12 servicios ambientales y 5 perjuicios ambientales asociados a la AUP, además de 197 iniciativas en áreas donde existe un incentivo para la práctica, pero en áreas que deben ser protegidas de acciones humanas. Se concluye que la AUP tiene potencial para ofrecer benefícios ambientales, aunque puede ejercer presiones negativas sobre el medio ambiente.

\footnotetext{
* Autor correspondente em: Universidade Nove de Julho, PPG em Cidades Inteligentes e Sustentáveis, São Paulo, Brasil, 01504-001.

E-mail: amarilislcfgallardo@gmail.com (Amarilis L. Gallardo, ORCID 0000-0002-5169-997X)
}

https://doi.org/10.47842/juts.v4i1.35

ISSN: 2675-780X 


\section{Introdução}

Por estar extremamente relacionada às condições do clima, a agricultura é um dos setores mais propensos a sofrer efeitos das mudanças climáticas que estão sendo discutidas e observadas nos últimos tempos. Estas mudanças apresentam efeitos diferentes nos sistemas naturais e humanos dependendo do nível de vulnerabilidade do sistema. Neste sentido, os países em desenvolvimento são os mais vulneráveis a essas mudanças do clima, podendo ser duramente atingidos pelos seus efeitos adversos. Para os países da América Latina, isso é reforçado pela sua economia fortemente dependente de recursos naturais diretamente ligados ao clima na agricultura, na geração de energia hidrelétrica, entre outros setores (MUDANÇA DO CLIMA, 2005).

Ao mesmo tempo, as práticas de agricultura tradicional podem potencializar esse fenômeno global. As práticas de Agricultura Urbana e Periurbana (AUP), baseadas em agroecologia, apresentam menor dependência de insumos externos à unidade de produção, diminuindo emissões e custo de produção (AQUINO e ASSIS, 2007) e levam a importantes ganhos ambientais, dentre eles: a reutilização dos resíduos orgânicos, aumento da biodiversidade, recuperação de áreas degradadas, criação de áreas verdes, influência positiva sob o sistema de drenagem (VALDIONES, 2013).

O Plano Diretor Estratégico (PDE) deve orientar o desenvolvimento do município, buscando definir estratégias de planejamento urbano para reorganizar a cidade com o objetivo de garantir a melhoria na qualidade de vida (PREFEITURA DE SÃO PAULO, 2015). A lei de Parcelamento, Uso e Ocupação do Solo (Lei 16.402/16, São Paulo/SP) classifica o uso do solo de acordo com as atividades compatíveis para o local, levantando em conta a ocupação já existente, interesses públicos, a infraestrutura disponível e os impactos da atividade.

O PDE prevê o zoneamento urbano como um dos principais instrumentos na gestão urbana. No PDE de São Paulo, a agricultura urbana está prevista como atividade de incentivo na Macroárea de Controle e Qualificação Urbana e Ambiental e na Macroárea de
Contenção Urbana e Uso Sustentável. As Zonas de Preservação e Desenvolvimento Sustentável (ZPDS) são porções do território destinadas à conservação da paisagem e à implantação de atividades econômicas compatíveis com a manutenção e recuperação dos serviços ambientais, dentre elas, a agricultura.

Em São Paulo, há diversas iniciativas, cada qual com a sua particularidade e capacidade de produção. Casos de sucesso como o projeto Ligue os Pontos e Cidades Sem Fome, mostram o quanto a atividade pode ser impactante, gerando benefícios ambientais, sociais e econômicos. Ambas as iniciativas se caracterizam pela criação de uma agricultura sustentável e resiliente que favorecem a criação de uma cidade mais justa.

A AUP, produção agrícola que ocorre no interior ou nas zonas periféricas da cidade e que está vinculada às dinâmicas da região onde ocorre, é vista neste contexto como aliada da agricultura rural para: (1) garantir o acesso da população a alimentos em quantidade e qualidade, (2) aumentar a resiliência das metrópoles pela promoção de serviços ambientais, e (3) promoção de empregos.

Este trabalho tem como principal objetivo discutir os principais serviços e desserviços ambientais associados à agricultura urbana e periurbana existentes no município de São Paulo. São estabelecidos como objetivos específicos: a) levantar as bases teóricas para análise dos serviços e desserviços ambientais associados às práticas de AUP; b) analisar a distribuição territorial das iniciativas existentes no município de São Paulo, relacionadas ao uso previsto no PDE e ao acesso a alimentos saudáveis pela população do município.

Considera-se que a discussão da expansão da AUP, em políticas públicas de planejamento urbano e outros incentivos, deve estar embasada em estudos que permitam o entendimento da sua capacidade de produção, das diferentes possibilidades e dos potenciais benefícios ou restrições. Para tanto, fazse necessário consolidar os dados disponíveis sobre a prática na cidade tanto em relação à distribuição das iniciativas existentes, quanto em termos de questões relevantes que devem ser consideradas no planejamento de novas iniciativas. 


\section{Referencial teórico}

O referencial teórico está assentado na apresentação do tema agricultura urbana e periurbana e do tema serviços ecossistêmicos e serviços ambientais.

\subsection{Agricultura Urbana e Periurbana (AUP)}

A agricultura é uma atividade imprescindível para a sociedade, tanto no abastecimento de alimentos quanto na questão econômica. Durante o processo de crescimento urbano, houve uma segregação entre as cidades e as áreas rurais, apesar da dependência mútua, também ocasionando uma separação de gestão e de políticas públicas rurais e urbanas (MASCARÓ, ACUNHA e PAGGOTTO, 2017).

Até o início do século XX, a agricultura aplicada em contextos urbanos era considerada, em geral, como uma forma de auxiliar a população a passar por momentos de crises, como aconteceu com a liberação de espaços públicos vagos para o cultivo de alimentos na cidade de Detroit, nos Estados Unidos, durante a crise conhecida como Pânico de 1893 (BIZERRA, 2016).

Entretanto, evidências históricas de práticas de agricultura nas cidades foram identificadas em várias partes do mundo e em diversas épocas desde o início da agricultura (MOUGEOT, 1994). Como colocado por Bizerra (2016), até meados do século XX, nota-se um crescimento das hortas urbanas fortemente relacionado a tempos de crise, com exemplos nos Estados Unidos e na Europa nos períodos das duas grandes guerras mundiais.

A partir da década de 1970, a agricultura urbana e periurbana (AUP) passou a ser fomentada por ativistas urbanos e utilizada principalmente para produção de alimentos, aumento da renda familiar, desenvolvimento de habilidades profissionais, expressão cultural, recreação, socialização e inclusão social (BIZERRA, 2016).

Atualmente, as práticas de agricultura urbana são mais frequentes e não necessariamente ligadas a momentos de crise. No Brasil, a partir da década de 1990, organizações da sociedade civil e de governos passaram a incentivar a organização e expansão das práticas após identificarem as melhorias na qualidade de vida da população atreladas às iniciativas de agricultura nas cidades (MOURA, FERREIRA e LARA, 2013).

Em relação aos conceitos de agricultura urbana e periurbana, de forma simplificada, quando ocorre a associação das práticas agrícolas, da economia e da ecologia com o espaço físico, a população que o habita e seu estilo de vida, tem-se a chamada Agricultura Urbana, que pode também ser chamada de Periurbana, caso esteja localizada em áreas limítrofes da cidade (BIZERRA, 2016).

A agricultura é um dos setores mais suscetíveis aos efeitos das mudanças climáticas. Os países em desenvolvimento são os mais vulneráveis a essas mudanças do clima, podendo ser duramente atingidos pelos seus efeitos adversos. Para o Brasil, isso é reforçado pela sua economia fortemente dependente de recursos naturais diretamente ligados ao clima na agricultura, na geração de energia hidrelétrica, entre outros setores (MUDANÇA DO CLIMA, 2005).

Neste contexto, a agricultura urbana e periurbana (AUP) vem como uma aliada da agricultura rural em relação ao abastecimento urbano, uma vez que diminui a distância entre produtor e consumidor, aumenta a oferta de alimentos e proporciona oportunidades de trabalho para pessoas desempregadas (BIZERRA, 2016). Além disso, as práticas de AUP baseadas em agroecologia apresentam menor dependência de insumos externos à unidade de produção, culminando no aumento da segurança alimentar das cidades (AQUINO e ASSIS, 2007).

A prática da AUP em cidades e metrópoles traz benefícios para a população, sobretudo para as que se encontram em condição de vulnerabilidade socioeconômica, pois contribui na garantia de segurança alimentar e permite a geração de empregos e renda, melhoria do bem-estar das famílias e diminuição de desigualdades de gênero.

Ganhos ambientais podem ser relacionados com a prática da atividade como a reutilização dos resíduos orgânicos, aumento da biodiversidade, recuperação de áreas degradadas, criação de 
áreas verdes, influência positiva sob o sistema de drenagem (VALDIONES, 2013). Os benefícios proporcionados pela recuperação de áreas na prática da AUP permitem que o homem obtenha serviços ambientais decorrentes do manejo empregado na área. Com a recuperação dos ecossistemas, serviços como sequestro de carbono, polinização, produção de alimentos, regulação de clima, controle de pestes e sensação de bem-estar são potencializados (LIN, PHILPOTT e JHA, 2015).

Vale ressaltar que apesar dos benefícios apresentados relacionados à prática da agricultura urbana e periurbana a atividade também pode ser uma fonte de desserviços, gerando impactos negativos para a região onde está implantada caso o manejo não seja adequado, por exemplo, aumentando a pressão sobre os recursos hídricos e gerando poluição do ar com aplicação de defensivos agrícolas. Entretanto, busca-se mostrar que a AUP pode ser uma alternativa de enfrentamento eficaz na garantia de melhores condições de qualidade de vida, segurança alimentar e promoção de serviços ambientais, com principal impacto nas populações mais pobres.

\subsection{Serviços Ecossistêmicos e Serviços Ambien- tais}

Serviços ecossistêmicos são os benefícios que o homem obtém dos ecossistemas (COSTANZA, D'ARGE, et al., 1997) (MILLENNIUM ECOSYSTEM ASSESSMENT, 2005), seja de forma direta ou indireta, consciente ou inconsciente (COSTANZA, GROOT, et al., 2017).

AAvaliação Ecossistêmica do Milênio (MILLENNIUM ECOSYSTEM ASSESSMENT, 2005) classifica esses serviços ecossistêmicos nas categorias: serviços de provisão, que provêm bens, tais como alimentos, água, fibras; serviços de regulação, que regulam aspectos do ecossistema, tais como controle de enchente, purificação do ar e água, polinização e controle de pestes; serviços culturais, que produzem benefícios de recreação, valor estético e religioso; e serviços de suporte, que dão suporte para que os outros serviços existam, tais como ciclagem de nutrientes e formação do solo.
Para alguns autores como Muridian (2009), os benefícios que o homem obtém da prática da agricultura urbana não seriam definidos como serviços ecossistêmicos, que são aqueles providos por ecossistemas exclusivamente naturais, mas como serviços ambientais, aqueles providos por ecossistemas manejados pelo homem. Para fins de definição, será adotada a terminologia de Muridian (2009), também de acordo com o PDE de São Paulo, que adota serviços ambientais como nomenclatura para tratar dos benefícios oriundos das áreas verdes urbanas. Vale destacar que a grande maioria da bibliografia utilizada trata o tema utilizando o conceito tradicional trazido por COSTANZA, D'ARGE, et al. (1997), sendo assim o termo serviços ecossistêmicos ainda estará presente em alguns trechos do trabalho quando acompanhado de referência.

De qualquer forma, a agricultura urbana tem-se mostrado uma grande detentora de biodiversidade, de vertebrados, invertebrados e plantas (LIN, PHILPOTT e JHA, 2015), o que possibilita a manutenção da provisão dos serviços ecossistêmicos (COSTANZA, GROOT, et al., 2017) (Loreau et al., 2001), inclusive aqueles valorizados por gestores urbanos, como escoamento de água de tempestades, purificação do ar, sequestro e armazenamento de carbono e provisão de água (MCLAIN et al., 2012 apud LIN, PHILPOTT e JHA, 2015).

Em revisão bibliográfica, Lin, Philpott e Jha (2015) citam também os serviços de polinização, controle de pragas e de regulação climática, todos oferecidos por espaços de agricultura urbana.

Lin, Philpott e Jha (2015) citam ainda alguns estudos que mostram possíveis trade-offs, que resultam de uma má gestão desses espaços: aumento da população de vetores de algumas doenças, como mosquitos; competição de espécie endógenas com espécies nativas, podendo levar ao aparecimento de pragas na flora e fauna; incremento de escoamento com componentes químicos e/ou tóxicos, quando o cultivo não é orgânico.

Por isso, cabe destacar, a importância de estudos que preencham a lacuna científica existente quanto 
aos serviços ambientais de maneira que o manejo dos espaços de agricultura urbana permita a sua intensificação e permitam melhores condições de produção de alimentos.

Dentro do contexto da agricultura urbana e os serviços ambientais que permitem a sua realização, vale destacar a capacidade de recuperação do solo e de fornecer serviços ambientais de provisão de alimentos, desde que passe por um manejo adequado que permita a sua reabilitação das condições de compactação e baixo nível de matéria orgânica, que geralmente se encontram em centros urbanos (BENISTON e LAL, 2012 apud LIN, PHILPOTT E JHA, 2015).

É nessa capacidade de recuperação e de fornecimento de alimentos que se considera que a agricultura urbana busca forças para a sua expansão, com a premissa de qualquer solo pode ser um solo produtivo, desde que bem manejado.

\section{Métodos}

\subsection{Levantamento de Serviços e Desserviços Ambientais da AUP}

Para o levantamento dos serviços e desserviços ambientais associados à AUP foi realizada uma revisão sistemática da literatura. Como alternativa para o levantamento de tais informações, artigos publicados em revistas científicas foram analisados como forma de obter um panorama do "estado da arte" sobre o tema.

A partir da definição das palavras-chave, a busca consistiu na procura de artigos no ScienceDirect, pois foi a fonte mais utilizada para a obtenção dos mesmos. Como a prática da AUP é mais disseminada em países da Europa e nos Estados Unidos, os artigos que tratam da temática e especialmente da sua relação com os serviços ecossistêmicos, tendem a se concentrar na língua inglesa e foram, consequentemente, o alvo das nossas buscas.

Como palavras-chave foram utilizadas: urban agriculture, ecosystem services, community garden, allotment, trade-offs, e as diversas combinações entre as palavras que pudessem nos indicar artigos que estavam relacionados com a prática de agricultura urbana e com os serviços ecossistêmicos/ambientais envolvidos, tendo como resultado 222 artigos. Além dos artigos encontrados na busca direta, alguns artigos da página de artigos recomendados também foram incluídos na base de dados. A seleção dos artigos foi realizada a partir da leitura do seu resumo e, quando necessário, também a conclusão para descartar artigos que não estavam diretamente relacionados ao escopo da pesquisa. A partir da seleção dos artigos, procurou-se identificar no texto quais serviços ambientais e possíveis trade-offs ou desserviços eram relacionados com a prática. Como resultado dessa etapa, foram selecionados 10 artigos lidos na íntegra. Como forma de melhor estruturar as informações obtidas, uma tabela apresenta os serviços ou desserviços encontrados, o tipo de serviço encontrado, descrição e a fonte utilizada como referência.

\subsection{Distribuição das iniciativas de AUP em São Paulo}

O mapa de iniciativas atuais no município de São Paulo foi feito com base nas informações disponibilizadas nos sites do Movimento Urbano de Agroecologia de São Paulo (MUDA-SP) e do Sampa+Rural, plataformas onde estão compilados locais que apresentam atividades de AUP.

A partir da coleta dos dados, a confecção do mapa foi feita no software QGis, que permite a visualização, edição e análise de dados georreferenciados. Também foram carregadas no programa camadas com os limites municipais do estado de São Paulo, o limite da cidade de São Paulo e os limites dos distritos da cidade, retirados do Geosampa e da plataforma de dados abertos da Prefeitura de São Paulo, assim como camadas com informações referentes a zona urbana, zona rural e macroáreas de desenvolvimento estabelecidas no Plano Diretor Estratégico de 2015.

Para análise da segurança alimentar e nutricional, foram compilados dados de equipamentos de abastecimento municipais para a elaboração de um terceiro mapa com a densidade de equipamentos de abastecimento (feiras livres, mercados e 
sacolões) por 10.000 habitantes nos distritos da cidade. Para isso, foram retirados do site do IBGE e do Geosampa os arquivos georreferenciados com informações de população (Censo 2010) e de equipamentos de abastecimento.

O mapa de iniciativas atuais foi feito com base nas informações disponibilizadas nos sites do Movimento Urbano de Agroecologia de São Paulo (MUDA-SP) e do Sampa+Rural, plataformas onde estão compilados locais com hortas, organizações relacionadas à agricultura urbana, restaurantes que servem pratos com alimentos orgânicos e feiras de produtos orgânicos. Desses, apenas os locais com hortas foram selecionados para compor o estudo do trabalho, uma vez que são os locais onde de fato há a produção dos alimentos e maior oferta dos serviços ambientais levantados.

Além disso, para basear uma análise integrada com a questão da segurança alimentar e nutricional, foram compilados dados de equipamentos de abastecimento municipais para a elaboração de um terceiro mapa com a densidade de equipamentos de abastecimento (feiras livres, mercados e sacolões) por 10.000 habitantes nos distritos da cidade. Para isso, foram retirados do site do IBGE e do Geosampa os arquivos georreferenciados com informações de população (Censo 2010) e de equipamentos de abastecimento, que também foram tratados no QGis para a produção do mapa final. A escolha dos tipos de estabelecimentos analisados foi realizada com base no estudo de Duran (2013), que identificou as feiras, os mercados e os sacolões municipais como os equipamentos que apresentam o maior acesso a alimentos saudáveis.

\section{Resultados e discussão}

Os resultados e discussão são apresentados quanto à avaliação dos serviços ambientais, dos desserviços ambientais e da análise espacial das AUP em São Paulo.

\subsection{Análise potencial da prestação de serviços ambientais pela AUP}

Ao analisar os serviços ambientais encontrados envolvendo a agricultura urbana, pode-se concluir que a sua presença muito está relacionada com o manejo e a escala da atividade em estudo, pois o manejo tem a capacidade de proporcionar as condições necessárias para o surgimento/ intensificação de um determinado serviço e a escala da atividade dá uma ideia da complexidade que pode ser atingida dentro daquele sistema. Com isso, há de se notar que nem todos os serviços ambientais estão presentes em todas as iniciativas de AUP, pois cada uma tem suas características e isso pode favorecer ou não a presença de um determinado serviço, entretanto, como visto na bibliografia de análise, modelos semelhantes tendem a proporcionar os mesmos benefícios.

Em sua maioria, os modelos de agricultura urbana encontrados nos artigos analisados eram modelos de hortas comunitárias localizadas na região urbana da cidade e de allotments (loteamentos), na região periférica. De maneira geral, os modelos de AUP encontrados nos artigos são semelhantes aos encontrados em São Paulo, no que tange à sua localização, ocupação do solo e comunidade envolvida. Desse modo, é possível associar os potenciais benefícios e desserviços encontrados nos artigos com as características das atividades inseridas em São Paulo (Tabela 1). Em São Paulo, as atividades localizadas na região central apresentam características mais comunitárias e as atividades na região periférica da cidade apresentam maiores dimensões e capacidade de produção mais elevada.

\subsection{Análise dos trade-offs envolvidos na prática da AUP}

Apesar dos benefícios encontrados em artigos e observados na prática relacionados à prática da agricultura urbana, alguns aspectos negativos também foram identificados quando o manejo utilizado não era o adequado (Tabela 2).

Dentre os desserviços encontrados, a pressão sobre os recursos hídricos foi sem dúvida um dos mais notáveis. O contexto de crise hídrica apresentado por Lin et al. (2019), tem uma certa relação com o vivido por São Paulo em 2014, e que se desenha novamente em 2021, que passou por um racionamento de água intenso. Outro aspecto 
Tabela 1. Serviços ambientais relacionados à prática de AUP.

\begin{tabular}{|c|c|c|c|}
\hline Serviço ambiental & Tipo & Descrição & Referência \\
\hline Ciclo de nutrientes & Suporte & $\begin{array}{c}\text { Estudos mostram que os solos de hortas urbanas apresentaram ganho } \\
\text { na atividade microbiológica, promovendo a retenção de ciclagem de } \\
\text { nutrientes }\end{array}$ & \multirow{2}{*}{$\begin{array}{l}\text { Ines Cabral et al } \\
\text { (2017) }\end{array}$} \\
\hline $\begin{array}{l}\text { Permeabilidade do } \\
\text { solo }\end{array}$ & Regulação & $\begin{array}{c}\text { A AU promove o aumento de áreas permeáveis na cidade, favorecendo } \\
\text { a retenção de água da chuva e impactando positivamente na drenagem } \\
\text { urbana }\end{array}$ & \\
\hline $\begin{array}{l}\text { Regulação da } \\
\text { qualidade do ar }\end{array}$ & Suporte & $\begin{array}{c}\text { A capacidade das plantas de atrair e absorver partículas e moléculas } \\
\text { favorece a melhora da qualidade do ar }\end{array}$ & \multirow[t]{2}{*}{$\begin{array}{l}\text { Menconi et al } \\
\quad(2020)\end{array}$} \\
\hline $\begin{array}{l}\text { Melhora do clima } \\
\text { local }\end{array}$ & Regulação & $\begin{array}{l}\text { A AU é uma atividade que pode influenciar positivamente na melhora do } \\
\text { clima local, através da alteração da cobertura do solo dentro das cidades }\end{array}$ & \\
\hline Polinização & Regulação & $\begin{array}{l}\text { A AUP promove a recuperação e ganho de biodiversidade, promovendo } \\
\text { um habitat favorável para seres vertebrados e invertebrados }\end{array}$ & \multirow{4}{*}{$\begin{array}{l}\text { Brenda B. Lin } \\
\text { and Monika H. } \\
\text { Egerer (2019) }\end{array}$} \\
\hline Controle de pestes & Regulação & $\begin{array}{c}\text { A AU promove a recuperação de áreas degradadas e de terrenos } \\
\text { abandonados, promovendo o controle de pestes e manejo adequado } \\
\text { nesses locais }\end{array}$ & \\
\hline $\begin{array}{l}\text { Recuperação da } \\
\text { qualidade do solo }\end{array}$ & Suporte & $\begin{array}{l}\text { A AUP promove a recuperação da qualidade do solo, impactando } \\
\text { positivamente na retenção de carbono e retenção de água }\end{array}$ & \\
\hline $\begin{array}{l}\text { Produção de } \\
\text { alimentos }\end{array}$ & Provisão & $\begin{array}{c}\text { Ao gerar alimentos, a AUP favorece a segurança alimentar da } \\
\text { comunidade onde está localizada, pois facilita o acesso à alimentos } \\
\text { saudáveis }\end{array}$ & \\
\hline $\begin{array}{l}\text { Troca de } \\
\text { conhecimento }\end{array}$ & Cultural & $\begin{array}{c}\text { A AUP proporciona a troca e valorização de conhecimentos entre as } \\
\text { pessoas envolvidas na atividade }\end{array}$ & \\
\hline $\begin{array}{l}\text { Regulação da } \\
\text { qualidade do ar }\end{array}$ & Suporte & $\begin{array}{c}\text { A capacidade das plantas de atrair e absorver partículas e moléculas } \\
\text { favorece a melhora da qualidade do ar }\end{array}$ & $\begin{array}{l}\text { Menconi et al } \\
\quad(2020)\end{array}$ \\
\hline $\begin{array}{l}\text { Senso de } \\
\text { comunidade }\end{array}$ & Cultural & $\begin{array}{c}\text { A AUP promove o estreitamento de relações entre a comunidade } \\
\text { envolvida (vizinhos, amigos, parentes), sendo um dos principais } \\
\text { benefícios observados no estudo }\end{array}$ & Aerts et al \\
\hline Bem estar & Cultural & $\begin{array}{c}\text { A melhora do bem estar, relacionado a prática de atividades físicas e } \\
\text { redução do estresse, foi algo citado como um benefício percebido pelos } \\
\text { agricultores que participaram do estudo }\end{array}$ & 120101 \\
\hline
\end{tabular}

relacionado a São Paulo, é a presença de áreas contaminadas que, se forem ocupadas por esse tipo de atividade, podem gerar impactos negativos.

\subsection{Distribuição territorial das atividades de AUP no município}

A coleta dos dados disponíveis nos mapas do MUDA-SP e do Sampa+Rural permitiu a compilação de 197 pontos com práticas de AUP na cidade de São Paulo, sendo 176 localizadas em regiões urbanas e 21 em regiões rurais, como pode ser observado na Figura 1.

Em relação às macroáreas estabelecidas no PDE, é possível observar na Tabela 3 que, na zona urbana, já existem diversas iniciativas de AUP implantadas em áreas onde não há o incentivo direto à prática. A maioria (56\%) está localizada em macroáreas onde foi identificado um incentivo indireto, ou seja, não explicitamente indicado no Plano, mas áreas que foram definidas com objetivos que também podem ser alcançados com a prática da AUP, como uso não residencial do espaço urbano para geração de empregos e renda.

Ao mesmo tempo, as macroáreas que possuem um incentivo explícito à prática de AUP no PDE são as que possuem menos iniciativas mapeadas, o que demonstra que apenas esse incentivo não é suficiente para que a prática seja mais difundida e implantada pela cidade.

Essa distribuição mostra que a disseminação da prática não depende, necessariamente, do incentivo direto de políticas públicas, uma vez que iniciativas organizadas pela própria sociedade civil são implantadas no ambiente urbano mesmo sem esse apoio. Desta forma, a falta de políticas públicas voltadas para a prática da agricultura no ambiente das cidades não se mostra um empecilho à disseminação da prática, embora a criação e 
Tabela 2. Trade-offs relacionados à prática de AUP.

\begin{tabular}{|c|c|c|}
\hline Desserviço & Descrição & Referências \\
\hline $\begin{array}{l}\text { Pressão sobre os } \\
\text { recursos hídricos }\end{array}$ & $\begin{array}{l}\text { O consumo de água nas hortas urbanas pode gerar uma maior pressão sobre os } \\
\text { recursos hídricos, principalmente em épocas de seca, o que pode ocasionar uma } \\
\text { competição com o abastecimento urbano }\end{array}$ & $\begin{array}{l}\text { Brenda B. Lin and } \\
\text { Monika H. Egerer } \\
\text { (2019) }\end{array}$ \\
\hline $\begin{array}{l}\text { Contaminação de } \\
\text { alimentos }\end{array}$ & $\begin{array}{c}\text { A atividade de AUP em áreas com solo contaminado ou próximo a vias de tráfego } \\
\text { intenso podem influenciar negativamente na qualidade e segurança do alimento } \\
\text { produzido para a saúde humana, devido a poluição urbana }\end{array}$ & $\begin{array}{l}\text { G. Caneva et al } \\
(2020)\end{array}$ \\
\hline $\begin{array}{c}\text { Utilização de } \\
\text { defensivos agrícolas }\end{array}$ & $\begin{array}{c}\text { O estudo destaca a possibilidade de utilização de defensivos agrícolas em atividades } \\
\text { não orgânicas. A utilização desses químicos no ambiente urbano tem o potencial de } \\
\text { levar a contaminação do solo e/ou do ar }\end{array}$ & $\begin{array}{c}\text { Brenda B. Lin et al } \\
\text { (2015) }\end{array}$ \\
\hline $\begin{array}{c}\text { Perda de } \\
\text { biodiversidade }\end{array}$ & $\begin{array}{c}\text { A prática da AUP em locais de vegetação nativa pode implicar na perda de } \\
\text { biodiversidade, em decorrência da intensidade do manejo e da substituição de } \\
\text { vegetação natural }\end{array}$ & $\begin{array}{c}\text { Ines Cabral et al } \\
(2017)\end{array}$ \\
\hline $\begin{array}{l}\text { Perda de beleza } \\
\text { cênica }\end{array}$ & $\begin{array}{l}\text { O estudo destaca a perda de beleza cênica como consequência da perda de } \\
\text { vegetação natural na região periurbana da cidade }\end{array}$ & $\begin{array}{l}\text { Marta Sylla et al. } \\
\text { (2020). }\end{array}$ \\
\hline
\end{tabular}

implantação adequada de políticas pudesse tornar seu planejamento mais estratégico e integrado ao desenvolvimento do município.

Além disso, também é possível notar pela Tabela 3 que existem iniciativas em áreas de preservação de ecossistemas naturais, onde predominam remanescentes florestais naturais, ecossistemas importantes para a manutenção da biodiversidade e conservação do solo, várzeas e nascentes

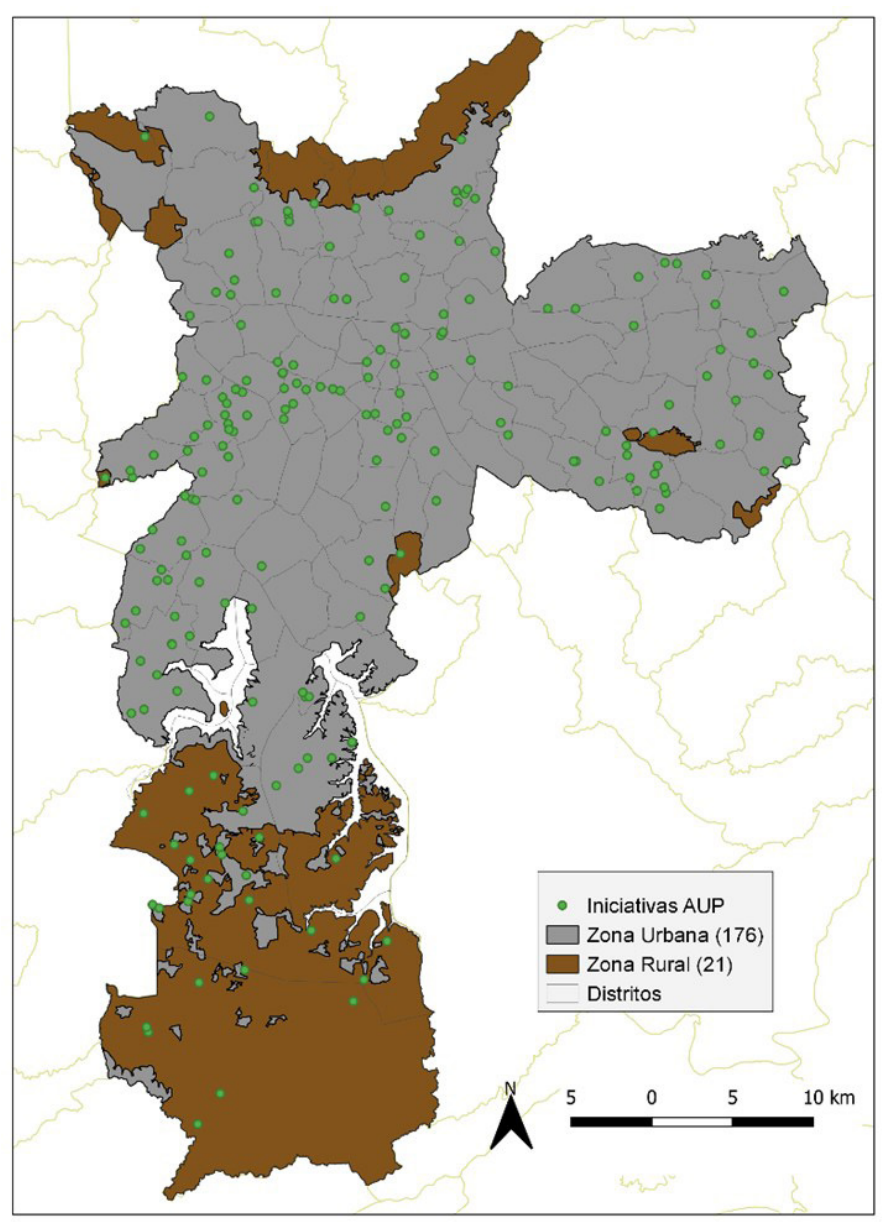

Fig. 1. Iniciativas de AUP no município de São Paulo. de cursos d'água ainda pouco impactadas por atividades antrópicas (PREFEITURA DE SÃO PAULO, 2015). Embora as iniciativas nessas áreas sejam poucas, a prática da agricultura pode exercer grande pressão sobre os ecossistemas naturais, principalmente no que se refere ao uso de água, perda de biodiversidade e perda de beleza cênica e, portanto, é importante que se tenha um cuidado para que não haja uma disseminação da prática nessas áreas.

Em relação à segurança alimentar e nutricional, foi avaliada a disponibilidade de equipamentos de abastecimento na cidade de São Paulo, levandose em conta estabelecimentos municipais (feiras, mercados e sacolões). A Figura 2 permite visualizar de forma mais quantitativa a desigualdade no acesso a equipamentos de abastecimento que provém alimentos saudáveis, de forma que as regiões centrais chegam a apresentar até três vezes mais estabelecimentos disponíveis do que as áreas periféricas. Isso mostra a importância e o potencial que a AUP tem em garantir segurança alimentar e nutricional a essa população, pois garante acesso a alimentos saudáveis, frescos e a uma dieta mais variada.

\section{Considerações finais e recomendações}

Pela revisão do estado da arte e dos casos presentes em São Paulo, a AUP promove uma diversidade de serviços ambientais na cidade, impactando positivamente as áreas ao seu redor. Dentre esses serviços ambientais destacados na 
Tabela 3. Quantidade de iniciativas de AUP por macroárea do Plano Diretor Estratégico.

\begin{tabular}{|c|c|c|c|}
\hline Incentivo PDE 2015 & Macroárea & Iniciativas & $\%$ \\
\hline \multirow{2}{*}{ Direto (19\%) } & Macroárea de contenção urbana e uso sustentável & 17 & $9 \%$ \\
\hline & Macroárea de controle e qualificação urbana e ambiental & 20 & $10 \%$ \\
\hline \multirow{3}{*}{ Indireto (56\%) } & Macroárea de estruturação metropolitana & 36 & $18 \%$ \\
\hline & Macroárea de redução da vulnerabilidade urbana & 33 & $17 \%$ \\
\hline & Macroárea de redução da vulnerabilidade urbana e recuperação ambiental & 41 & $21 \%$ \\
\hline \multirow{3}{*}{$\begin{array}{c}\text { Não relacionado } \\
(25 \%)\end{array}$} & Macroárea de preservação dos ecossistemas naturais & 4 & $2 \%$ \\
\hline & Macroárea de qualificação da urbanização & 20 & $10 \%$ \\
\hline & Macroárea de urbanização consolidada & 26 & $13 \%$ \\
\hline
\end{tabular}

literatura, os serviços culturais, em decorrência da troca de conhecimento e bem-estar, e a segurança alimentar são os benefícios de maior destaque para a sociedade, alinhando-se ao encontrando em São Paulo. No município de São Paulo, a AUP está representada, em sua maioria, por hortas comunitárias mantidas por moradores da região ou associações sociais, contribuindo para a interação social e geração de emprego e renda de diversas pessoas, além de proporcionar acesso a alimentos saudáveis, como é o caso das iniciativas que estão

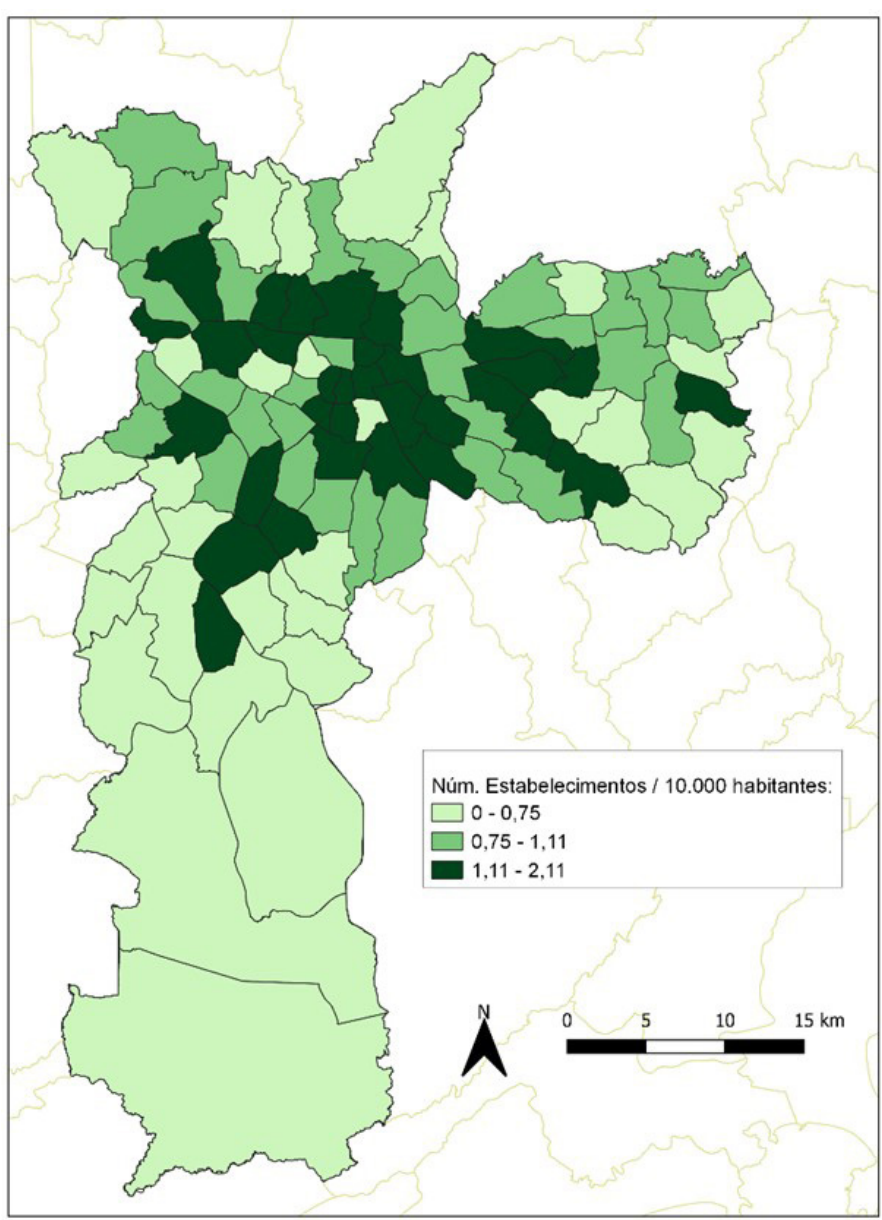

Fig. 2. Densidade de equipamentos de abastecimento por distrito. presentes em escolas e bairros menos favorecidos.

Ainda, por promover serviços como regulação do clima local e aumento da permeabilidade do solo, a AUP pode contribuir para o aumento da resiliência do município em relação a eventos extremos decorrentes das mudanças do clima, assim como proposto no PNA. Entretanto, vale ressaltar que essa temática pode ser explorada por estudos complementares sobre a relação da AUP e os potenciais serviços ambientais identificados na revisão bibliográfica, pois muitos estudos são de percepção ambiental, podendo ser utilizadas ferramentas de engenharia para mensurar real influência da agricultura urbana sobre a oferta de serviços ambientais urbanos.

Em relação aos desserviços encontrados na literatura e que têm forte relação com momentos vividos em São Paulo, é o cenário apresentado por Lin et al. (2019) na Califórnia, onde as atividades apresentavam como principal trade-off a pressão sobre os recursos hídricos. São Paulo, em momentos recentes, passou por um episódio de estresse hídrico e uma possível expansão das atividades de AUP poderia ocasionar um aumento da demanda de água, portanto é importante que medidas para redução da água no setor. Nesse contexto, destacam-se as medidas adotadas pela ONG Cidades Sem Fome, onde a água de chuva é captada e armazenada para utilização em hortas como uma maneira de amenizar a intensidade da demanda de água para irrigação das plantações.

Os resultados desse trabalho contribuem para um maior embasamento científico sobre a AUP como uma estratégia de adaptação às mudanças climáticas, destacando benefícios como que 
englobam regulação do clima local e aumento da permeabilidade do solo, tornando as áreas urbanas mais resilientes aos eventos extremos.

Em relação à situação atual da AUP no município de São Paulo, foi possível levantar um número considerável das práticas existentes e avaliar as regiões em que ocorrem de acordo com o que é previsto no Plano Diretor Estratégico do município. Porém, nem todas as iniciativas existentes foram mapeadas devido à falta de dados específicos fornecidos pelas plataformas consultadas, como endereços ou coordenadas geográficas, principalmente de iniciativas próprias localizadas na área rural do município. Para um levantamento e acompanhamento mais preciso da abrangência da AUP no município de São Paulo, seria de grande valia que as plataformas do MUDA-SP e do Sampa+Rural integrassem suas informações e fornecessem uma base georreferenciada de dados e completa das iniciativas cadastradas.

O diagnóstico e análise das AUP no município de São Paulo, as iniciativas mapeadas consistem, em sua maioria, em hortas comunitárias, que podem ser tanto de bairro quanto de instituições específicas, escolas, associações sociais ou de espaços culturais de vivência e aprendizado. Além das hortas, também foram mapeados alguns sítios, onde a produção ocorre em maior escala. Nota-se que as hortas estão mais concentradas na área urbana do município, a maioria em macroáreas do PDE que não recebem um incentivo direto à implantação da prática. Por sua vez, os sítios estão mais concentrados na área rural, mais especificamente na região Sul, onde o PDE estabelece tanto macroáreas em que há o incentivo direto para o desenvolvimento de AUP, quanto macroáreas destinadas à proteção dos recursos naturais, onde, em teoria, a AUP pode exercer grande pressão e, consequentemente, trazer impactos negativos.

O que pode ser observado é que as atividades que se encontram na área urbana da cidade têm maior capacidade de proporcionar os benefícios levantados na literatura em termos de serviços ambientais, devido às características da região onde são implantadas, geralmente terrenos vazios que, ao serem recuperados, trazem benefícios como o aumento da permeabilidade do solo, o envolvimento da comunidade do entorno e a produção de alimentos para essa comunidade. Na região periférica do município, onde as atividades trazem os principais benefícios de geração de emprego e renda, o risco ambiental associado é maior devido à necessidade de proteção dos mananciais e das áreas com vegetação nativa, então é importante que as atividades sejam melhora avaliadas em termos de potenciais desserviços ambientais. Embora o PDE incentive de forma explícita a prática de AUP nas macroáreas de Controle e Qualificação Urbana e Ambiental e de Contenção Urbana e Uso Sustentável, as iniciativas presentes nessas áreas correspondem a apenas 19\% das totais mapeadas, o que permite concluir que o tema não é tão abordado no Plano como poderia ser. Dados os benefícios potenciais e a presença de mais de 50\% das iniciativas já existentes em áreas onde o Plano propõe objetivos que podem ser atingidos com a prática da AUP, como o uso não residencial do espaço urbano para ampliar a geração de emprego e renda e reduzir a distância entre a moradia e o trabalho. Desta forma, a revisão do PDE poderia incorporar diretrizes que orientassem a implantação da AUP de forma a potencializar os benefícios e prevenir os impactos negativos associados à prática de acordo com as características de cada macroárea, garantindo um manejo adequado para cada situação. É importante que haja um cuidado nas áreas urbanas quanto à contaminação dos alimentos por conta da qualidade do ar e do solo e, nas áreas rurais, com a pressão sobre os recursos naturais, principalmente água e vegetação nativa.

O acesso a alimentos saudáveis e de qualidade é muito desigual dentro do município de São Paulo, onde as regiões Norte e Sul apresentam significativamente menos estabelecimentos disponíveis para a população adquirir alimentos naturais. Nota-se que os distritos de médio e alto nivel socioeconômico apresentam cerca de três vezes mais estabelecimentos disponíveis para a aquisição de alimentos naturais do que os de baixo nível socioeconômico. Nesta situação, a implantação de iniciativas de AUP em regiões com menor densidade de equipamentos de abastecimento 
pode contribuir para o acesso de uma população de menor nível socioeconômico a alimentos de qualidade, contribuindo para a segurança alimentar e nutricional, assim como gerar oportunidades de emprego e renda.

Conclui-se que esse trabalho contribui para um aprofundamento do conhecimento sobre situação atual da AUP no município de São Paulo, destacando os potenciais benefícios e impactos negativos associados à prática e quanto à distribuição das regiões. Esse trabalho representa uma contribuição inicial para compor um referencial teórico para a formulação de políticas públicas relacionadas ao tema e para que a implantação de novas iniciativas seja feita de realizada de modo estratégico e eficiente.

\section{Agradecimentos}

A terceira autora agradece à Fapesp, por meio do processo Fapesp 19/18988-9, e ao CNPq, por meio do processo CNPa 303542/2020-9, pelo apoio ao desenvolvimento dessa pesquisa.

\section{Referências bibliográficas}

AERTS, R.; DEWAELHEYNS, V.; ACHTEN, W. M. J. Potential ecosystem services of urban agriculture: a review. PeerJ, v. 4, 2016.

AQUINO, A. M. D.; ASSIS, R. L. D. Agricultura orgânica em áreas urbanas e periurbanas com base na agroecologia. Ambiente \& Sociedade, v. X, n. 1, (2007) p. 137-150.

BIZERRA, L. V. A. D. S. Agricultura urbana e periurbana: revisão bibliográfica sobre a situação atual brasileira. 2016. Monografia (Bacharelado em Agronomia) - Universidade de Brasília, Brasília, 2016.

CANEVA, G.; CICINELLI, E.; SCOLASTRI, A.; BARTOLI, F. Guidelines for urban community gardening: Proposal of preliminary indicators for several ecosystem services (Roma,e, Italy). Urban Forestry \& Urban Greening, v. 56 (2020), p. 126866.
CABRAL, I.; KEIM, J.; ENGELMANN, R.; KRAEMER, R.; SIEBERT, J.; BONN, A. Ecosystem services of allotment and community gardens: A Leipzig, Germany case study. Urban Forestry \& Urban Greening, v. 23 (2014), p. 44-53.

COSTANZA, R. et al. The value of the world's ecosystem services and natural capital. Nature, 15 (1997), p. 253-260.

COSTANZA, R. et al. Twenty years of ecosystem services: How far have we come and how far do we still need to go? Ecosystem Services, v. 28 (2017), p. 1-16.

LIN, B. B.; PHILPOTT, S.; JHA, S. The future of urban agriculture and biodiversity-ecosystem services: Challenges and next steps. Basic and Applied Ecology, v. 16, Issue 3 (2015), p. 189-201.

LIN, B. B.; EGERER, M. Global social and environmental change drives the management and delivery of ecosystem services from urban gardens: A case study from Central Coast, California. Global Environmental Change-human and Policy Dimensions, v. 60 (2020), p. 102006.

MENCONI, M. E.; HELAND, L.; GROHMANN, D. Learning from the gardeners of the oldest community garden in Seattle:Resilience explained through ecosystem services analysis. Urban Forestry \& Urban Greening, v. 56 (2020), p. 126878.

MILLENNIUM ECOSYSTEM ASSESSMENT. Ecosystems and Human Well-being: General Synthesis. Island Press. Washington, 2005.

MOURA, J. A.; FERREIRA, W. R.; LARA, L. D. B. L. S. Agricultura Urbana e Periurbana. Mercartor Revista de Geografia da UFC, v. 12, n. 27 (2013), p. 13.

MUDANÇA DO CLIMA. Negociações internacionais sobre a mudança do clima: vulnerabilidade, impactos e adaptação à mudança do clima. Núcleo de Assuntos Estratégicos da Presidência da República (NAE). Brasília, p. 250. 2005. 
MUDA-SP. Movimento Urbano de Agroecologia de

São Paulo, 2020. Disponivel em: <http://muda.org. br>. Acesso em: maio 2020.

PREFEITURA DE SÃO PAULO. Plano Diretor Estratégico do Município de São Paulo, 2015. Disponível em: <https://www.prefeitura.sp.gov.br/ cidade/secretarias/urbanismo/legislacao/plano_ diretor/index.php?p=201105>. Acesso em: Junho 2020.

SYLLA, M.; HAGEMANN, N.; SZEWRANSKI, S. Mapping trade-offs and synergies among periurban ecosystem services to address spatial policy. Environmental Science \& Policy, v. 112 (2020), p. 79-90.

VALDIONES, A. P. G. Panorama da agricultura urbana e periurbana no município de São Paulo. 2013. Dissertação (Mestrado em Mudança Social e Participação Política) - Escola de Artes, Ciências e Humanidades, Universidade de São Paulo, São Paulo, 2013. 